\title{
Identification of a IncRNA-associated competing endogenous RNA-regulated network in clear cell renal cell carcinoma
}

\author{
BEIBEI LIU*, TANTU MA*, QINGWEN LI, SHENG WANG, WEI SUN, \\ WENYONG LI, JIANMIN LIU and YUANYUAN GUO
}

Department of Urology, The First Affiliated Hospital of Bengbu Medical College, Bengbu, Anhui 233000, P.R. China

Received October 19, 2018; Accepted April 4, 2019

DOI: $10.3892 / \mathrm{mmr} .2019 .10290$

\begin{abstract}
Long noncoding RNAs (lncRNAs) act as competing endogenous RNAs (ceRNAs) in the regulation of gene expression in various physiological and pathological processes. The present study aimed to explore the IncRNA-miRNA-mRNA interactions in clear cell renal cell carcinoma (ccRCC) using comprehensive bioinformatics analysis. RNA-seq data were downloaded from the TCGA Data Portal, and the differentially expressed lncRNAs (DElncRs), miRNAs (DEmiRs), and mRNAs (DEGs) between tumoral and control samples were identified using the edgeR package. The correlations between the DemiR/DElncR expression levels and clinical features were evaluated using nonparametric regression analysis. The Kaplan-Meier method was used to identify DElncRs associated with overall survival time. Then, the DElncR-DEmiR interaction pairs were predicted using miRcode and the starBase v2.0 database, and DEmiR-DEG pairs were predicted using the miRTarBase database. Then, a ceRNA-regulated network of ccRCC was constructed based on these interactions. Genes in the network were also assigned to functional categories in the KEGG pathway database. A total of 1,573 DEGs, 37 DelncRs, and 62 DEmiRs were identified. Moreover, several DElncRs were significantly associated with patient clinical variables; for example, TCL6 was significantly associated with tumor grade and AJCC pathological stage. Next, 38 pairs of DElncR-DEmiR interactions (13 DElncRs and 8 DEmiRs) were identified. Among the 8 DEmiRs that target DElncRs, six were found to target DEGs. Based on the identified DElncR-DEmiR interactions
\end{abstract}

Correspondence to: Dr Jianmin Liu or Dr Yuanyuan Guo, Department of Urology, The First Affiliated Hospital of Bengbu Medical College, 287 Mayor Huai Road, Bengbu, Anhui 233000, P.R. China

E-mail: liu_john_jm@sina.com

E-mail: bb_dynasty123@163.com

\section{*Contributed equally}

Key words: clear cell renal cell carcinoma, competing endogenous RNAs, IncRNA-miRNA-mRNA interactions and DEmiR-DEG interactions, a ceRNA-regulated network comprising 203 nodes and 221 edges was constructed (with MIC >0.15 and MIC- $\mathrm{p}^{2}>0.15$ ). The novel lncRNAs, DGCR5, MYCNOS, and PART1 may participate in the progression of ccRCC through cytochrome P450-mediated drug metabolism.

\section{Introduction}

Renal cell carcinoma (RCC) is the one of the 10 most common cancers, accounting for 2-3\% of all adult malignancies (1). Clear cell renal cell carcinoma (ccRCC) is the most common subtype of RCC, and approximately $75-80 \%$ of patients with RCC are diagnosed with ccRCC (2). Current therapies for renal cancer include conventional chemotherapy and radiation, however, the prognosis is poor (3). Therefore, to aid in the development of effective treatments for ccRCC, it is necessary to investigate the underlying molecular mechanism involved in the pathogenesis of ccRCC.

Only $2 \%$ of RNAs encode proteins in human cells. Many transcripts that do not encode proteins have recently been identified in large-scale genomic studies $(4,5)$. Although these RNAs are not translated, they play critical roles in regulating transcriptional and non-transcriptional processes (6). These non-coding RNAs (ncRNAs) can be broadly classified as small ncRNAs (<200 nucleotides, including microRNAs (miRNAs), siRNAs, and piRNAs) or long non-coding (lncRNAs) (>200 nucleotides). miRNAs are the most widely studied subclass of small ncRNAs, and they can post-transcriptionally regulate the expression of multiple genes via the imperfect complementarity to their target mRNA transcripts (7). miRNAs have been found to be involved in tumorigenesis, and evaluating the changes in miRNA expression could provide useful information to better understand tumor formation and progression (8). lncRNAs are eukaryotic RNAs with no coding capacity. Alteration of IncRNA expression has been reported to be associated with tumor development (9), and certain lncRNAs have been used as cancer biomarkers and potential targets in several types of tumors (10).

IncRNAs have been revealed to act as competing endogenous RNAs (ceRNAs), where the lncRNAs bind to and keep miRNAs from their cognate mRNA targets $(11,12)$. Hence, the mRNAs can be prevented from mediating miRNA-mediated repression $(11,12)$. A previous study indicated that small concentration changes in miRNA-mRNA or miRNA-ceRNA 
pairs can substantially affect the regulation of the gene expression network (13). For example, the lncRNA HULC has been previously shown to act as an endogenous sponge and inhibit miR-372, which plays an important role in tumorigenesis in liver cancer (14). The muscle-specific lnRNA, linc-MDI, regulates muscle differentiation by sequestering miR-133 and miR-135 and thus modulating the expression of MAML1 and MEF2C (15). Recent advances in the study of the functions and mechanisms of lncRNAs in physiological and pathological processes have led to expanding the possible manner in which lncRNAs function within the already complex system of miRNA-mediated gene regulation.

Although the expression profile of mRNA and lncRNA transcripts in ccRCC has been explored and several lncRNAs, such as HIF-1alpha-AS1, H19, KCQN1OT1, and MALAT1, have been reported to be involved in renal cancer (16-18), the regulation mechanism of IncRNA-miRNA-mRNA in ccRCC has not been elucidated. In this study, we downloaded the data for an important cohort encompassing more than 300 samples from the Cancer Genomes Atlas (TCGA) and used a bioinformatics method to predict the regulation mechanism of IncRNAs-miRNAs-mRNAs in ccRCC.

\section{Materials and methods}

\section{Data}

RNA-seq data collection and processing. The TCGA database is a comprehensive, publicly available, and vast source of information on cancer genetic and epigenetic profiles. The TCGA RNA-seq expression datasets (level 3) for ccRCC were downloaded from the TCGA Data Portal (https://tcga-data. nci.nih.gov/tcga/) (June 3, 2016), which contained 606 mRNA expression profiles and 326 miRNA expression profiles. Since TCGA level 3 data typically represents aggregated, normalized, and/or segmented data, the level 3 data downloaded was analyzed without normalization. Based on the barcodes, the RNA-seq samples were matched to miRNAs, and a total of 326 samples, including 254 ccRCC samples and 71 normal controls, were obtained.

lncRNA and mRNA sequencing was performed on the Illumina Hiseq 2000 platform, and miRNAs were sequenced on the RNASeqV2 platform (https://www.cancer. gov/about-nci/organization/ccg/research/structural-genomics/ tcga/using-tcga/technology/Illumina-HiSeq2000-Data-Sheet). RNA-seq reads were processed using RNA-Seq by Expectation-Maximization (RSEM). In addition, IncRNAs and $\mathrm{mRNAs}$ were annotated and identified on the website of the HUGO gene nomenclature committee (HGNC, http://www. genenames.org/), which contains annotation information on 2,775 lncRNAs and 19,004 protein-coding genes.

Analysis of differentially expressed mRNAs (DEGs), IncRNAs (DElncRs), and miRNAs (DEmiRs). The differential expression of mRNAs, lncRNAs, and miRNAs was analyzed using the edgeR package (19), which is an R Bioconductor package that provides statistical routines for examining the differential expression of replicated count data in RNA-seq data. This package is based on an overdispersed Poisson model (20) to account for the variability at both the biological and technical levels. In addition, the Empirical Bayes methods (21) in this package were used to moderate the overdispersion across transcripts, improving the reliability of inferences. The nominal P-values were estimated based on the method of Benjamini and Hochberg (22), using the multtest package (http://www. bioconductor.org/packages/release/bioc/html/multtest.html) in R. Only RNAs with a false-discovery rate (FDR) $<0.05$ and IlogFC (fold change) $>1$ l were selected as DEGs, DelncRs, or DEmiRs.

Correlation analysis between the clinical characteristics and expression of DemiRs and DElncRs. The clinical characteristics of patients with ccRCC were extracted from the downloaded data, including sex (female vs. male), age at diagnosis ( $\geq 61$ years vs. $<61$ years), tumor grade (G3/G4 vs. G1/G2), tumor status (tumor-burden state vs. tumor-free state), American Joint Committee on Cancer pathologic stage (III/IV vs. I/II), tumor metastasis (yes vs. no), and overall survival time. Then, the DElncRs/DemiRs that were significantly correlated with these clinical characteristics were identified using nonparametric regression analysis. A survival curve was constructed using the Kaplan-Meier method (23), and comparisons using the log rank test were performed between patients with upregulated DElncRs and those with downregulated DElncRs.

Construction of a ceRNA-regulated network. The lncRNA targets of human miRNAs were downloaded from the miRCode database (http://www.mircode.org/), a comprehensive searchable map of putative miRNA target sites across the complete GENCODE annotated transcriptome established by Jeggari et al (24), and starBase v2.0 (http://starbase.sysu. edu.cn/), a platform comprised of 111 CLIP-Seq data sets and focusing on protein-lncRNA, protein-sncRNA, protein-mRNA and protein-pseudogene interactions. The miRNA targets from the miRTarBase (http://mirtarbase.mbc.nctu.edu.tw), which is the most updated collection of miRNA-target interactions, were identified.

According to the identified miRNA-lncRNA interactions and miRNA-mRNA interactions, a comprehensive lncRNA-miRNA-mRNA network was constructed. Then, the interactions in the IncRNA-miRNA-mRNA network were further identified by calculating maximal information coefficients (MICs) with cut-off values of MIC $>0.15$ and $\mathrm{MIC}-\mathrm{p}^{2}>0.15$.

Functional enrichment of DEGs in the ceRNA-regulated network. The DEGs in the ceRNA-regulated network were assigned to functional categories in the Kyoto Encyclopedia of Genes and Genomes (KEGG) pathway database (25) (https://www.genome.jp/kegg/), and the pathways significantly associated with ccRcc were obtained. Enrichment P-values were calculated using Fisher's exact tests using the equation below. Then, the functional pathways in the ceRNA-regulated network in which the DEGs were enriched were identified.

$$
p=1-\sum_{i=0}^{x-1} \frac{\left(\begin{array}{l}
M \\
i
\end{array}\right)\left(\begin{array}{l}
N-M \\
K-i
\end{array}\right)}{\left(\begin{array}{l}
N \\
K
\end{array}\right)}
$$

Here, $\mathrm{N}$ represents the number of genes in the whole genome; $\mathrm{M}$ represents the number of genes enriched in KEGG 

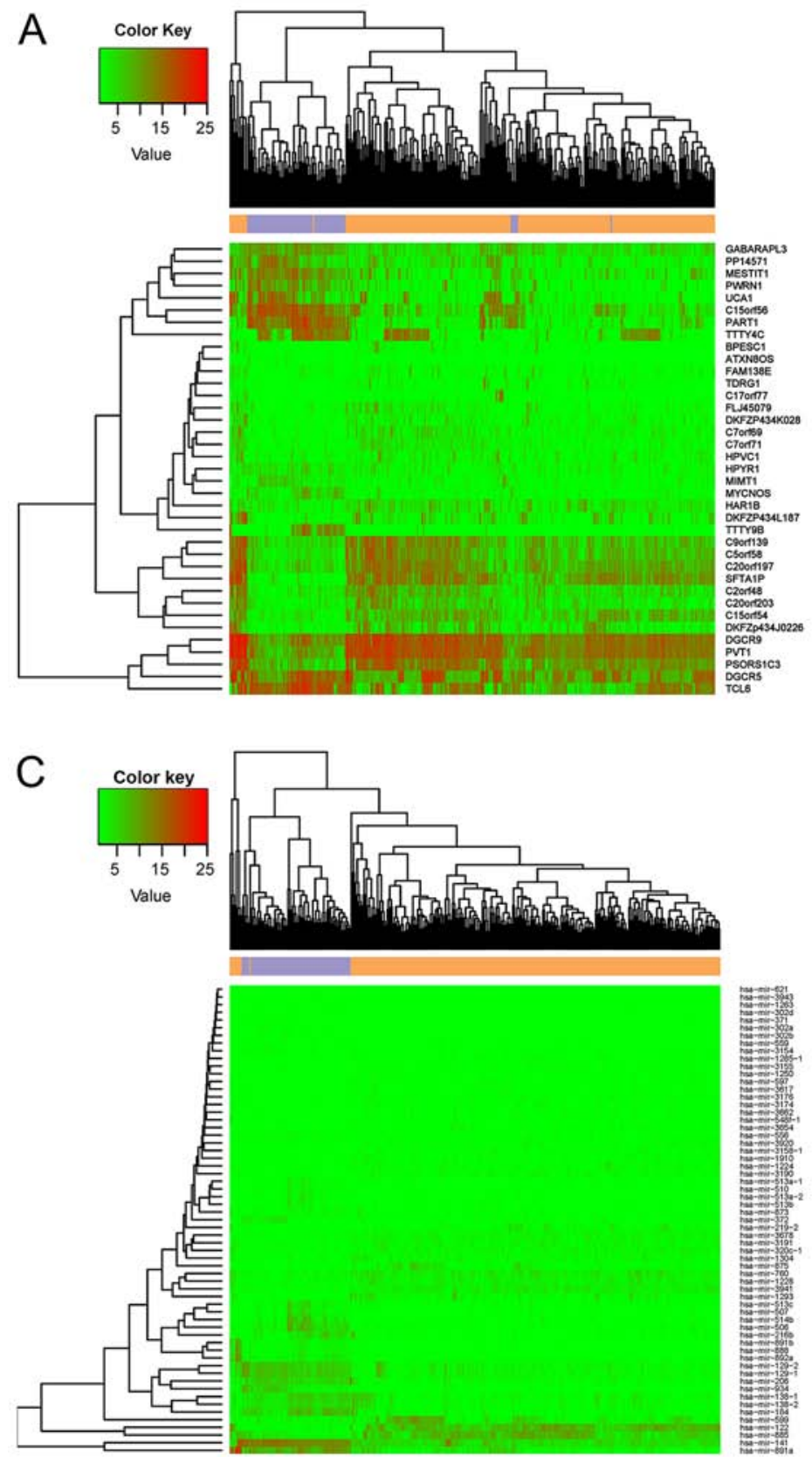

B
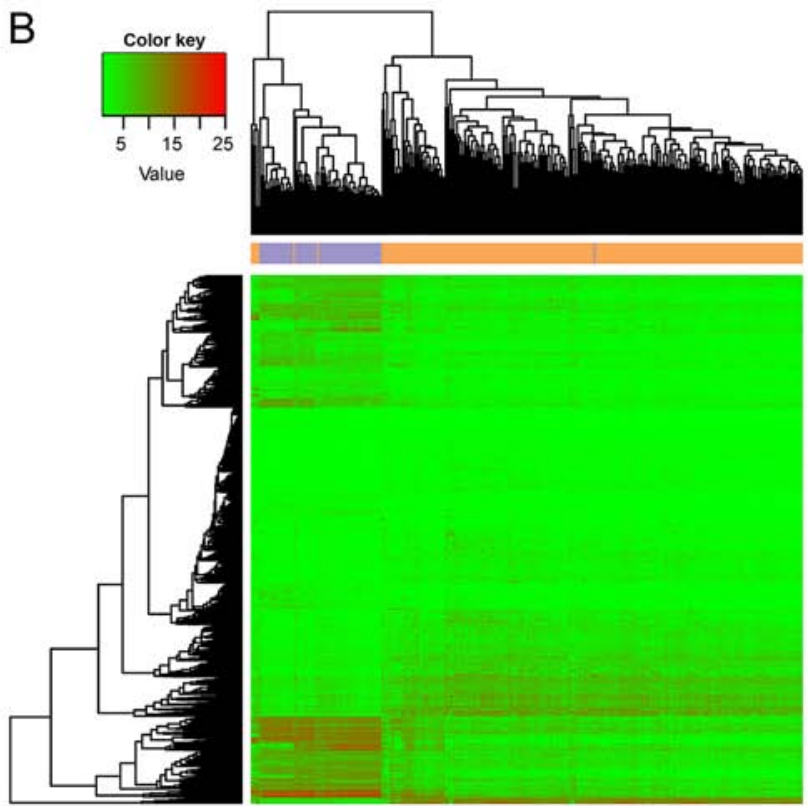

Figure 1. Heatmap representing the expression of differentially expressed (A) lncRNAs, (B) mRNAs, and (C) miRNAs. lncRNAs, long noncoding RNAs; miRNAs, microRNAs.

pathways; and $\mathrm{K}$ represents the number of differentially expressed genes. The Fisher's score represents the probability that at least $\mathrm{x}$ genes of $\mathrm{K}$ differentially expressed genes are enriched in KEGG pathways.

\section{Results}

Identification of DEGs, DelncRs, and DEmiRs. IncRNAs and mRNAs were annotated and identified on the HUGO gene nomenclature committee website. A total of 819 lncRNAs and 18,137 protein-coding transcripts were identified. Subsequently, using an FDR statistic $<0.05$ and $l \log \mathrm{FC}_{2}$ (fold change) $>11$, the differential expression analysis identified 1,573 DEGs and 37 DElncRs in the RNA-seq data and 62 DEmiRs in the miRNA-seq data. A heatmap representing the expression of the identified DElncRs, DEGs, and DEmiRs is presented in Fig. 1. The ccRCC samples were obviously separated from the control samples, suggesting that the DEGs represent a large difference between the tumor and control samples.

Identification of DemiRs and DelncRs that are significantly correlated with clinical characteristics. The correlation analysis between the clinical characteristics of patients and DemiRs indicated that the expression level of hsa-mir-216b was associated with two clinical variables, sex and tumor grade (Table I).

Moreover, the correlation between the clinical characteristics of patients and the expression of identified DelncRs was explored. The results are presented in Table II. The expression of DGCR9 and TTTY4C was significantly 
Table I. The interactions between DEmiRs and the clinical characteristics of patients.

Related miRNAs

Comparisons

Sex (female vs. male)

Age at diagnosis $(\geq 61$ vs. $<61)$

Tumor grade (G3 + G4 vs. G1 + G2)

Tumor status (with tumor vs. tumor-free)

AJCC pathological stage (III + IV vs. I + II)

Metastatic (yes vs. no)

\begin{tabular}{ll}
\multicolumn{2}{c}{ Related miRNAs } \\
\hline \multicolumn{1}{c}{ Upregulated } & \multicolumn{1}{c}{ Downregulated } \\
$\begin{array}{l}\text { hsa-mir-1293, hsa-mir-3654, } \\
\text { hsa-mir-1285-1 }\end{array}$ & hsa-mir-875, hsa-mir-216b, hsa-mir-599 \\
& $\begin{array}{l}\text { hsa-mir-513c, hsa-mir-1228, hsa-mir-3678, } \\
\text { hsa-mir-514b, hsa-mir-513b } \\
\text { hsa-mir-216b, hsa-mir-891b }\end{array}$ \\
$\begin{array}{l}\text { hsa-mir-3943, hsa-mir-510 } \\
\text { hsa-mir-934 } \\
\text { hsa-mir-891a, hsa-mir-3174 } \\
\text { hsa-mir-599, hsa-mir-888, } \\
\text { hsa-mir-1263 }\end{array}$ & $\begin{array}{l}\text { hsa-mir-507, hsa-mir-371 } \\
\text { hsa-mir-141 }\end{array}$ \\
\end{tabular}

DEmiRs, differentially expressed miRNAs; miRNAs, microRNAs.

Table II. The correlation between DElncRNAs and patient clinical features.

Identified DElncRNAs

Patient clinical characteristics

Upregulated

Downregulated

Sex (female vs. male)

Age at diagnosis ( $\geq 61$ vs. $<61$ years)

Tumor grade (G3/G4 vs. G1/G2)

Tumor status (tumor-burden state vs. tumor-free state)

AJCC pathological stage (III/IV vs. I/II)

Metastasis (yes vs. no)

$\begin{array}{ll}\text { C20orf203, HAR1B, DGCR9, DGCR5, } & \text { TTTY4C, TTTY9B } \\ \begin{array}{l}\text { FAM138E } \\ \text { C2orf48, DGCR9, DGCR5 }\end{array} & \text { ATXN8OS } \\ \text { PSORS1C3, C7orf71, DKFZP434K028, } & \text { HPYR1, TCL6 } \\ \text { FAM138E } & \\ \text { C7orf71, HAR1B, C2orf48, TDRG1 } & \text { TTTY4C } \\ \text { C7orf71, PSORS1C3, DKFZP434K028, } & \text { MYCNOS, TCL6 } \\ \text { FAM138E } & \\ \text { BPESC1, C20orf203, C7orf71, C2orf48, } & \text { MYCNOS, PP14571, TTTY4C, } \\ \text { DGCR9, DGCR5, DKFZp434J0226 } & \text { PART1 }\end{array}$
DGCR9, DGCR5, DKFZp434J0226
PART1

DElncRNAs, differentially expressed lncRNAs.

correlated with three clinical variables, sex, tumor status, and metastasis. TCL6 was revealed to be significantly associated with tumor grade and AJCC pathological stage. In addition, tumor metastasis was related to the upregulated expression of BPESC1, C20orf203, C7orf71, C2orf48, DGCR9, DGCR5, and DKFZp434J0226 and the downregulated expression of MYCNOS, PP14571, TTTY4C, and PART1. These results revealed certain candidate lncRNAs for ccRCC, such as C7orf71, C2orf48, DGCR9, DGCR5, and TTTY4C, and also suggested a sex difference in the stage distribution of ccRCC.

Kaplan Meier analysis was conducted on all the 37 DElncRs that were significantly associated with patient clinical characteristics. As a result, 7 DelncRs that were significantly associated with overall survival time of patients with ccRCC were identified, TTTY4C, PSORS1C3, C2orf48, HPYR1, PWRN1, C15orf56, and PART1. All of these DElncRs were negatively correlated with patient overall survival, as patients with lower expression of these DElncRs had longer survival times (Fig. 2).

Identification of DElncR-DEmiR interaction pairs. Predictions using miRcode and starBase revealed a total of 353 pairs of DElncR-miRNA interactions and 38 pairs of DElncR-DEmiR interactions. An interaction network of 13 DElncRs and 8 DEmiRs was constructed (Fig. 3). miR-122, miR-129, and miR-216b targeted more lncRNAs than the other miRNAs. miR-122 had a relationship with 8 DElncRNAs, including MYCNOS, PART1, and PWRN1. miR-129 targeted 7 DElncRNAs, including DGCR5, HPYR1, MYCNOS, PART1, and PWRN1. miR-216b had 7 targets, BPESC1, DGCR5, FAM138E, HPYR1, PVT1, PWRN1, and SFTA1P.

Identification of DEmiR-DEG interaction pairs. As aforementioned, a total of $8 \mathrm{DEmiRs}$ were revealed to be DElncR-targeting miRNAs. Then, the DEmiR-mRNA 
A

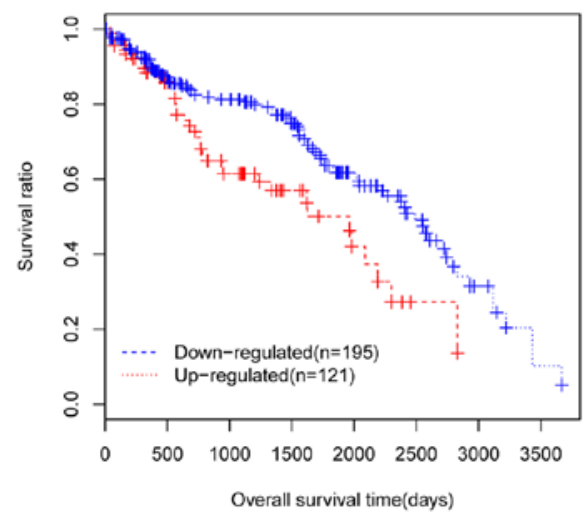

C

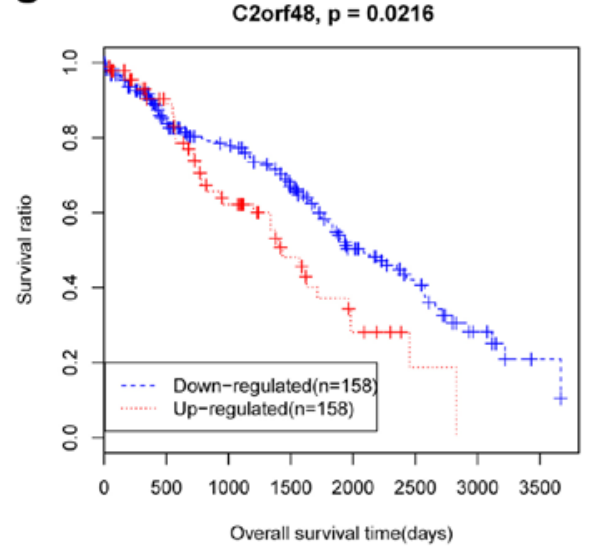

E

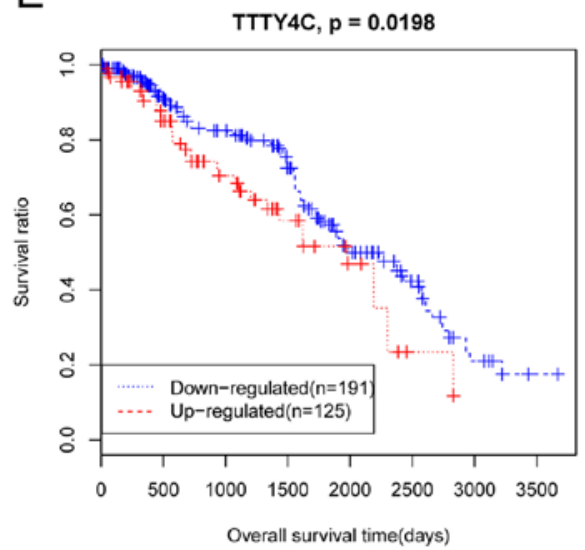

G

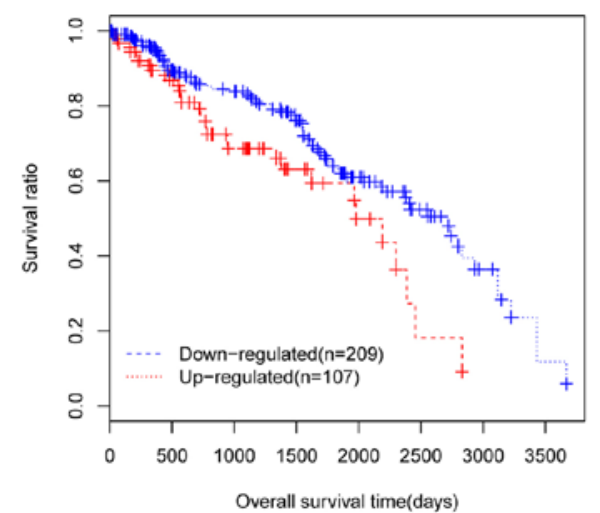

B

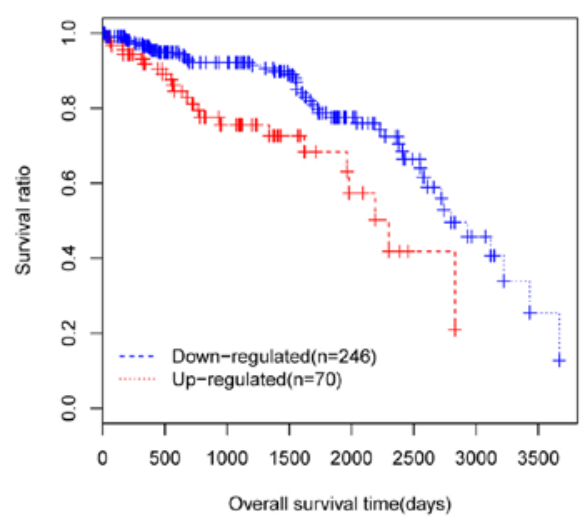

D

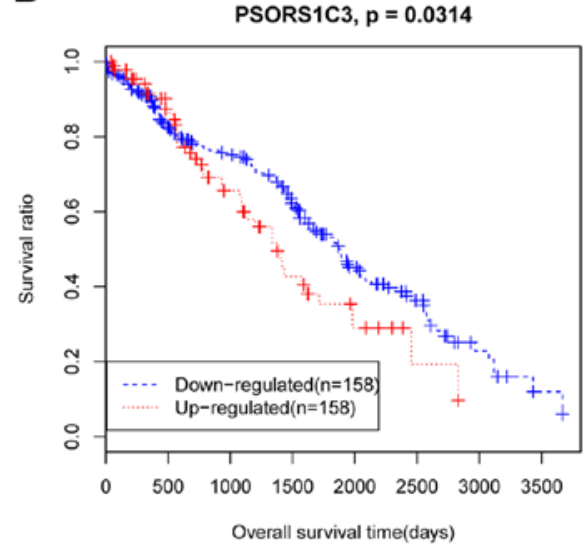

$\mathrm{F}$

PART1, $\mathrm{p}=0.0253$

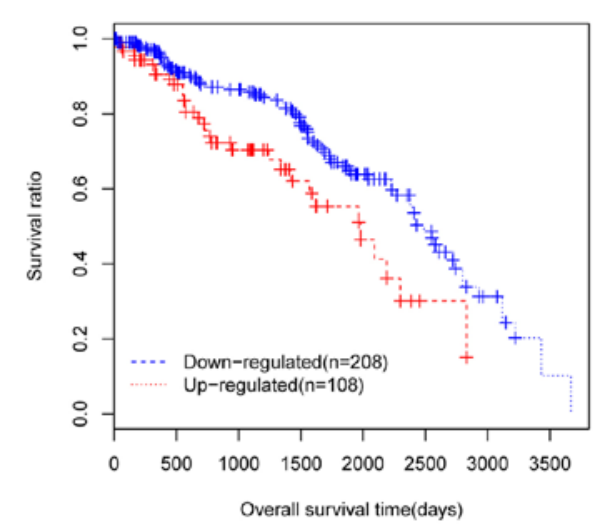

Figure 2. Kaplan-Meier survival curves for 7 identified differentially expressed lncRNAs, including (A) C15orf56, (B) HPYR1, (C) C2orf48, (D) PSORS1C3, (E) TTTY4C, (F) PART1, and (G) PWRN1. IncRNAs, long noncoding RNAs. 


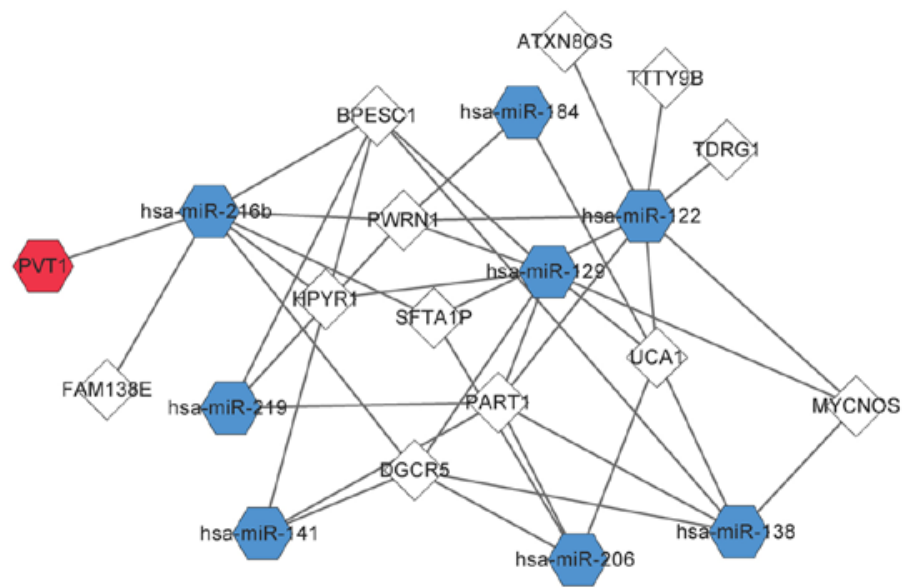

Figure 3. Interaction network of identified DElncRs and DEmiRs. The white diamonds represent differentially expressed lncRNAs; the blue hexagons represent differentially expressed miRNAs. The full lines represent the lncRNA-miRNA interactions. DElncRs, differentially expressed lncRNAs; DEmiRs, differentially expressed miRNAs; miRNAs, microRNAs. The red hexagon indicates a tumor-associated gene included in the allOnco database.

A

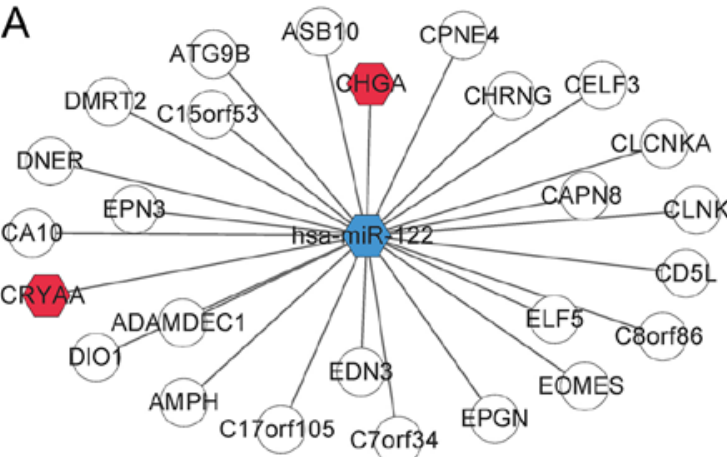

C

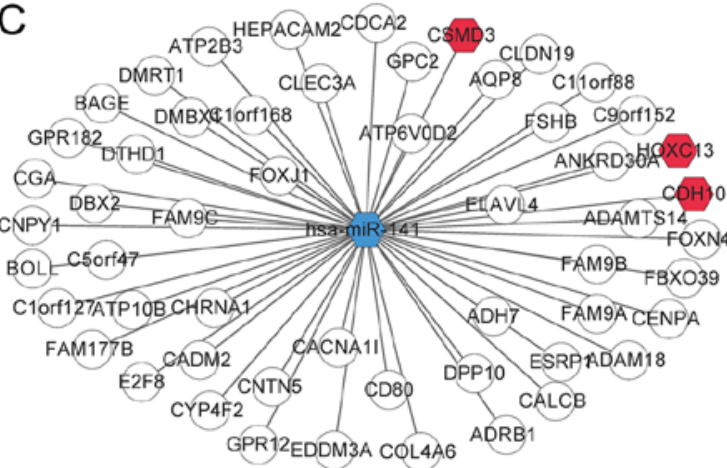

$E$

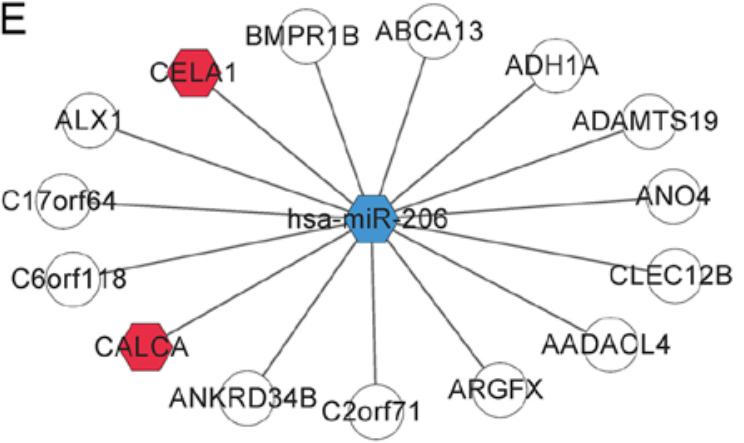

B
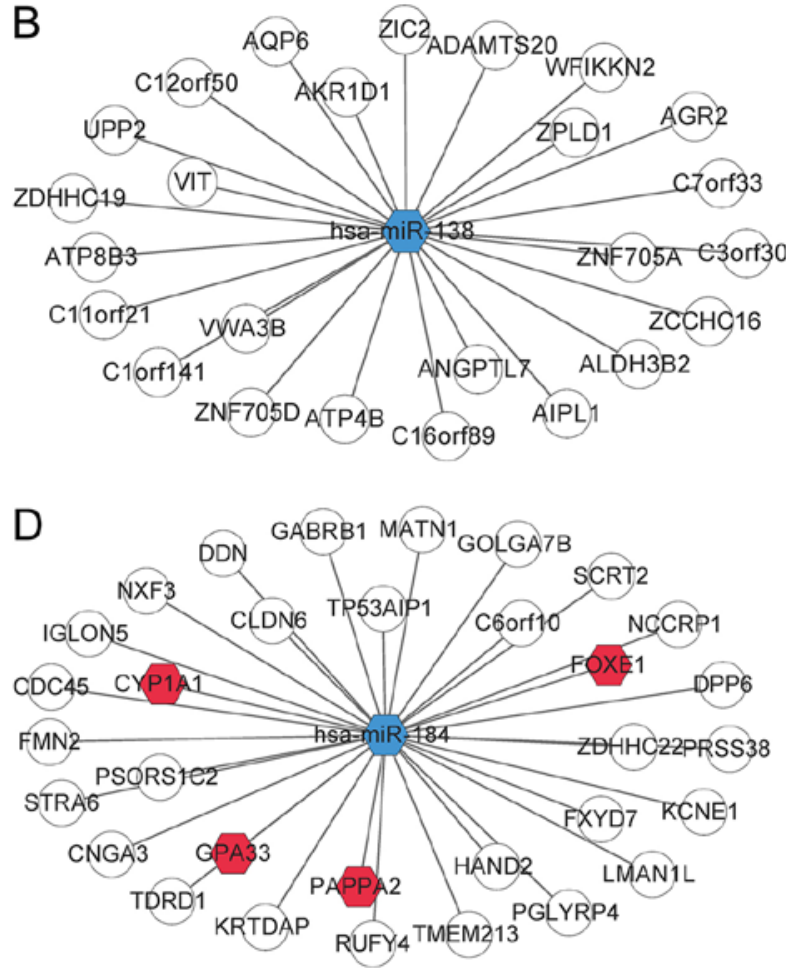

F

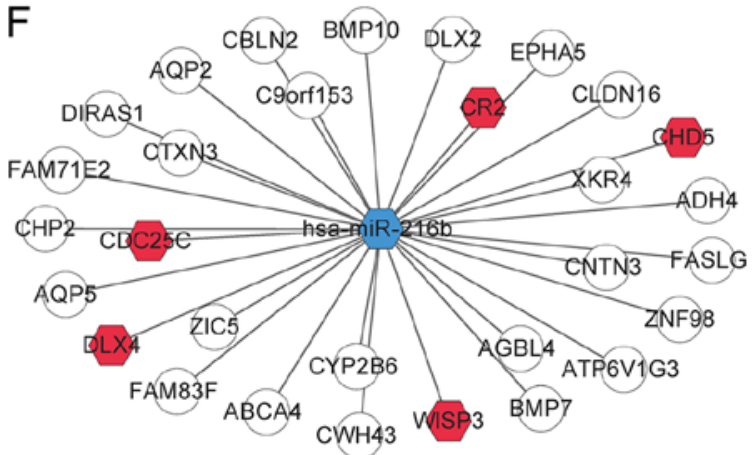

Figure 4. Interaction network of identified DEmiRs and DEGs. (A) miR-122-DEG interaction network; (B) miR-138-DEG interaction network; (C) miR-141-DEG interaction network; (D) miR-184-DEG interaction network; (E) miR-206-DEG interaction network; and (F) miR-216b-DEG interaction network. The blue hexagons represent differentially expressed miRNAs; the white circles represent differentially expressed genes; the red hexagons represent cancer-related genes from the allOnco database. The full lines represent the mRNA-miRNA interactions. DEmiRs, differentially expressed miRNAs; DEGs differentially expressed genes; miRNAs, microRNAs. 
Table III. The DEmiRs-DEGs interaction pairs extracted from miRTarBase.

\begin{tabular}{|c|c|c|}
\hline DEmiRs & No. of targeting DEGs & DEmiRs targeting DEGs \\
\hline has-miR-122 & 26 & $\begin{array}{l}\text { ADAMDEC1, AMPH, ASB10, ATG9B, C15orf53, C17orf105, C7orf34, C8orf86, } \\
\text { CA10, CAPN8, CD5L, CELF3, CHGA, CHRNG, CLCNKA, CLNK, CPNE4, } \\
\text { CRYAA, DIO1, DMRT2, DNER, EDN3, ELF5, EOMES, EPGN, EPN3 }\end{array}$ \\
\hline has-miR-138 & 25 & $\begin{array}{l}\text { ADAMTS20, AGR2, AIPL1, AKR1D1, ALDH3B2, ANGPTL7, AQP6, ATP4B, } \\
\text { ATP8B3, C11orf21, C12orf50, C16orf89, C1orf141, C3orf30, C7orf33, UPP2, VIT, } \\
\text { VWA3B, WFIKKN2, ZCCHC16, ZDHHC19, ZIC2, ZNF705A, ZNF705D, ZPLD1 }\end{array}$ \\
\hline has-miR-141 & 54 & $\begin{array}{l}\text { ADAM18, ADAMTS14, ADH7, ADRB1, ANKRD30A, AQP8, ATP10B, ATP2B3, } \\
\text { ATP6V0D2, BAGE, BOLL, C11 orf88, C1 orf127, C1orf168, C5orf47, C9orf152, } \\
\text { CACNA1I, CADM2, CALCB, CD80, CDCA2, CENPA, CGA, CHRNA1, CLDN19, } \\
\text { CLEC3A, CNPY1, CNTN5, COL4A6, CSMD3, CYP4F2, DBX2, DMBX1, DMRT1, } \\
\text { DPP10, DTHD1, E2F8, EDDM3A, ELAVL4, ESRP1, FAM177B, FAM9A, FAM9B, } \\
\text { FAM9C, FBXO39, FOXJ1, FOXN4, FSHB, GPC2, GPR12, GPR182, HEPACAM2, } \\
\text { HOXC13 }\end{array}$ \\
\hline has-miR-184 & 32 & $\begin{array}{l}\text { C6orf10, CDC45, CLDN6, CNGA3, CYP1A1, DDN, DPP6, FMN2, FOXE1, } \\
\text { FXYD7, GABRB1, GOLGA7B, GPA33, HAND2, IGLON5, KCNE1, KRTDAP, } \\
\text { LMAN1L, MATN1, NCCRP1, NXF3, PAPPA2, PGLYRP4, PRSS38, PSORS1C2, } \\
\text { RUFY4, SCRT2, STRA6, TDRD1, TMEM213, TP53AIP1, ZDHHC22 }\end{array}$ \\
\hline has-miR-206 & 15 & $\begin{array}{l}\text { AADACL4, ABCA13, ADAMTS19, ADH1A, ALX1, ANKRD34B, ANO4, ARGFX, } \\
\text { BMPR1B, C17orf64, C2orf71, C6orf118, CALCA, CELA1, CLEC12B }\end{array}$ \\
\hline has-miR-216b & 30 & $\begin{array}{l}\text { ABCA4, ADH4, AGBL4, AQP2, AQP5, ATP6V1G3, BMP10, BMP7, C9orf153, } \\
\text { CBLN2, CDC25C, CHD5, CHP2, CLDN16, CNTN3, CR2, CTXN3, CWH43, } \\
\text { CYP2B6, DIRAS1, DLX2, DLX4, EPHA5, FAM71E2, FAM83F, FASLG, WISP3, } \\
\text { XKR4, ZIC5, ZNF98 }\end{array}$ \\
\hline
\end{tabular}

DEmiRs, differentially expressed miRNAs, DEGs, differentially expressed genes.

interactions were collected from the miRTarBase database, and 6 out of 8 DEmiRs were revealed to target DEGs: hsa-miR-122 targeted 26 DEGs, hsa-miR-216b targeted 30 DEGs, hsa-miR-141 targeted 54 DEGs, hsa-miR-206 targeted 15 DEGs, hsa-miR-138 targeted 25 DEGs, and hsa-miR-184 targeted 32 DEGs (Table III). Collectively, these data indicated that hsa-miR-122 and hsa-miR-216b may be important in ccRCC. In addition, hsa-miR-141 had the most targets, followed by hsa-miR-184, also indicating their crucial role in ccRCC. Among the 182 DEmiR targets, 130 DEGs were identified as cancer-related genes after comparison with the allOnco database (http://www.bushmanlab.org/links/genelists), a collection of 2,125 cancer-related genes. The DEmiR-DEG interaction network is presented in Fig. 4.

Construction and functional annotation of a ceRNA-regulated network. A comprehensive lncRNA-miRNA-mRNA network was established based on the identified DElncR-DEmiR interactions and DEmiR-DEG interactions. By calculating MICs, with $\mathrm{MIC}>0.15$ and MIC- $\mathrm{p}^{2}>0.15$, a ceRNA-regulated network comprised of 203 nodes and 221 edges was constructed (Fig. 5). This network indicated the roles of hsa-miR-141, hsa-miR-184, hsa-miR-122, hsa-miR-216b, hsa-miR-138, and hsa-miR-206 in the ceRNA-regulated network in ccRCC.

To study the function of DEGs in the ceRNA-regulated network, KEGG pathway and gene ontology (GO) catalogue annotation were performed. These DEGs were enriched in 8 KEGG pathways (Fig. 6A), such as metabolism of xenobiotics by cytochrome $\mathrm{P} 450$, retinol metabolism, and drug metabolism, and $17 \mathrm{GO}$ terms, such as intrinsic to membrane, extracellular region, extracellular region part, transcription factor activity, ion transport, and proteolysis (Fig. 6B).

\section{Discussion}

Human lncRNAs have been revealed to act as competitors of mRNAs for miRNA binding in RNA-RNA cross-talk networks and regulate mRNA expression at the post-transcriptional level. Although certain differentially expressed lncRNAs and mRNAs have been identified in ccRCC by expression profiling and a branch of the MALAT1/miR-200s/ZEB2 pathway, and the functional triplets consisting of lncRNAs, mRNAs, and miRNAs, have been proposed to regulate the progression of ccRCC, a systematic analysis of the ceRNA-regulated network in ccRCC has not been performed. To understand the roles of the ceRNA-regulated network in the pathology of ccRCC, a transcriptome-wide identification and characterization of differentially expressed RNAs, including lncRNAs, miRNAs, and mRNAs was performed, and a comprehensive lncRNA-miRNA-mRNA network was constructed based on identified lncRNA-miRNA interactions and miRNA-mRNA interactions downloaded from an online 


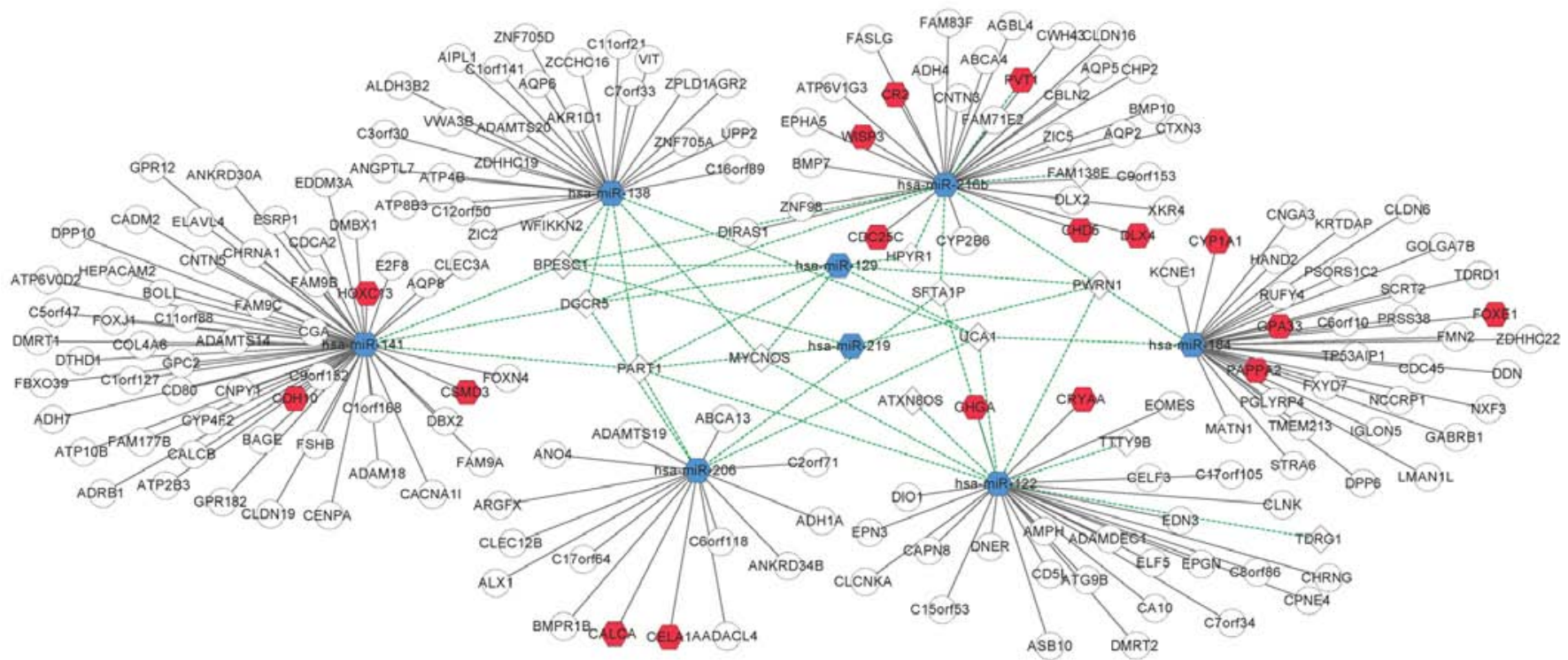

Figure 5. ceRNA-regulated network. The white diamonds represent differentially expressed lncRNAs; the blue hexagons represent differentially expressed miRNAs; the white circles represent differentially expressed genes; the red hexagons represent cancer-related genes from the allOnco database. The full lines represent the mRNA-miRNA interactions, and the dotted lines represent the lncRNA-miRNA interactions. ceRNAs competing endogenous RNAs; IncRNAs, long noncoding RNAs; miRNAs, microRNAs.

A

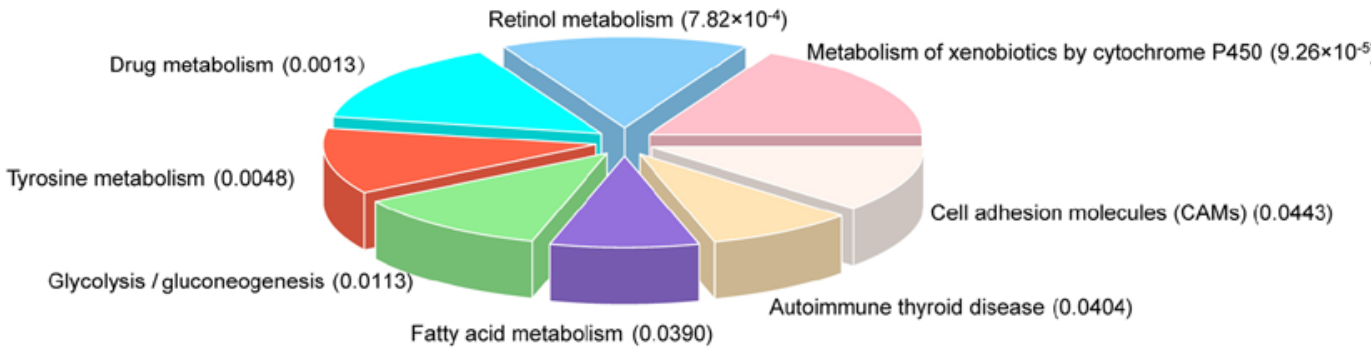

B

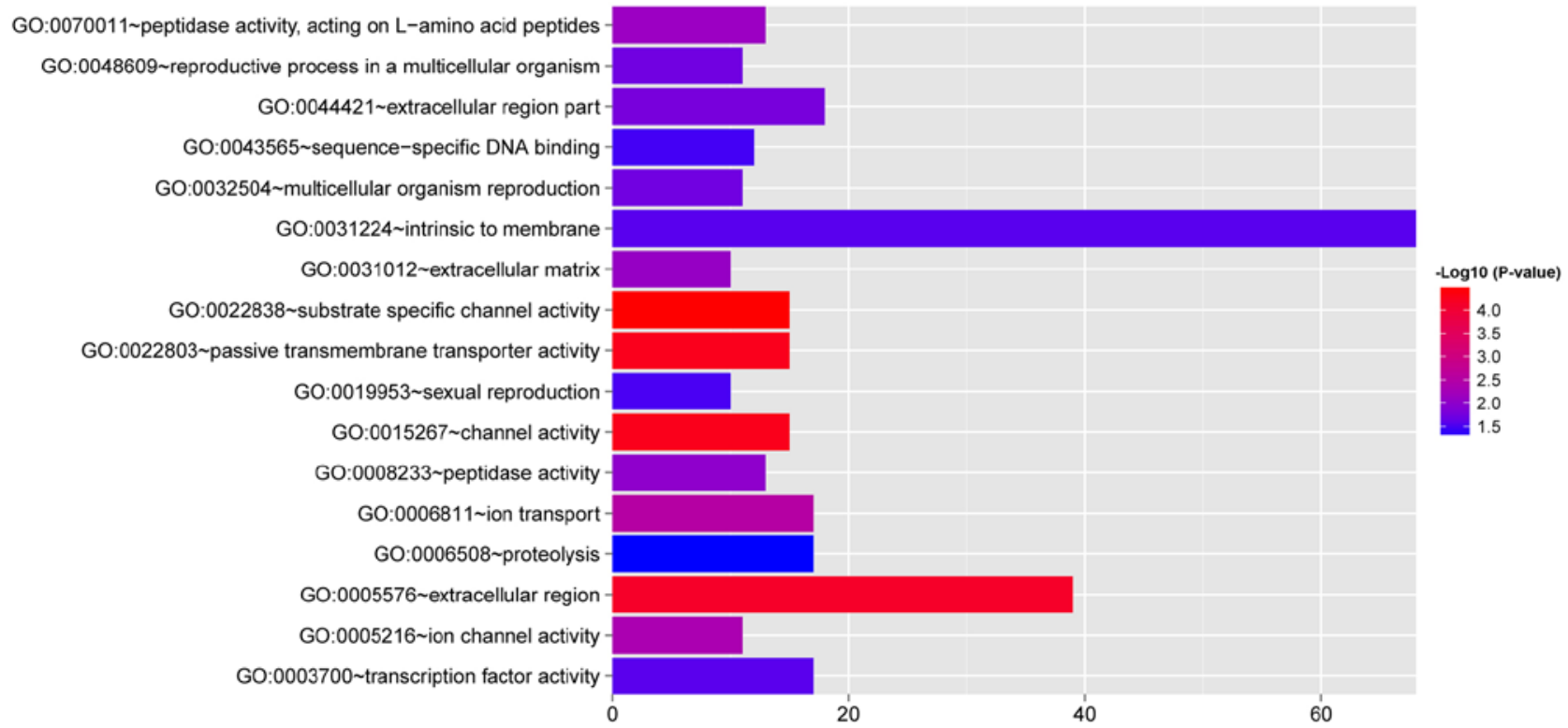

Figure 6. Involvement of the ceRNA-regulated network genes in (A) KEGG pathways and (B) GO terms.

data portal. The present study provides new insights that will aid in understanding the molecular mechanism involved in ccRCC.
To the best of our knowledge, the present study represents the first comprehensive description of the ceRNA-regulated network in ccRCC, as identified through the bioinformatics 
analysis of RNAseq data in a large patient cohort that encompasses 254 ccRCC tumor samples and 71 normal renal tissue samples. Several novel findings were obtained from our in-depth genomic analysis.

First, the expression of certain differentially expressed IncRNAs, suchas C2orf48,DGCR9,DGCR5,DKFZp434J0226, MYCNOS, PP14571, TTTY4C, and PART1, was correlated with tumor metastasis; moreover, the expression of TTTY4C, PSORS1C3, C2orf48, HPYR1, PWRN1, C15orf56, and PART1 was negatively correlated with patient overall survival. The expression level of miR-216b was revealed to be associated with sex and tumor grade in patients with ccRCC (26). Notably, TTTY4C and certain other lncRNAs were also revealed to be related to sex, which is consistent with the sex-dependent manner of ccRCC (27). All these identified lncRNAs have not been previously reported to be associated with ccRCC. DGCR5 has been revealed to be involved in the regulation of the proliferation, migration, and invasion of lung cancer (28) and lung adenocarcinoma (29). MYCNOS has been reported to cooperate with a crucial transcription factor, CCCTC-binding factor, to promote neuroblastoma progression through MYCN expression (30). PSORS1C3 has been demonstrated to be expressed in pluripotent and tumor cell lines (31). Therefore, further studies are required to clarify the role of these identified IncRNAs in ccRCC.

Second, a comprehensive IncRNA-miRNA-mRNA network in ccRCC was constructed, which revealed the roles of hsa-miR-141, hsa-miR-184, hsa-miR-122, hsa-miR-216b, hsa-miR-138, and hsa-miR-206 in the ceRNA-regulated network in ccRCC. The identified miRNAs in the ceRNA-regulated network, including miR-141 (32,33), miR-138 (32-34), miR-206c (35), miR-122 (36), miR-184 (37), and miR-216b (26), have been reported to play important roles in tumor progression in human ccRCC. In addition to these mRNAs, lncRNAs were also identified in the ceRNA-regulated network. As aforementioned, DGCR5 plays an important role in lung cancer $(28,29)$ and was revealed to target miR-216b, miR-129, miR-206, miR-141, and miR-138 in the present study. MYCNOS was revealed to target miR-129, miR-122, and miR-138. PART1 has also been revealed to target miR-129, miR-206, miR-219, miR-206, miR-141, and miR-138. Based on our results, the ceRNA-regulated network revealed how these competing modules (IncRNA-miRNA-mRNA) play a role in the pathogenesis of ccRCC and indicated the roles played by lncRNAs in the disease.

Moreover, the transcripts in the ceRNA network of ccRCC were revealed to be enriched in several pathways such as metabolism of xenobiotics by cytochrome P450, retinol metabolism, and drug metabolism and critical biological processes such as intrinsic to membrane, extracellular region, extracellular region part, transcription factor activity, ion transport, and proteolysis. These pathways share certain genes, such as ADH4 and ALDH3B2. Previous studies have reported that xenobiotic metabolism by cytochrome $\mathrm{P} 450$ and drug activation are involved in tumorigenesis in $\operatorname{RCC}(38,39)$.

Collectively, the use of bioinformatic methods can significantly improve our understanding of RNA communication in ccRCC at the transcriptome level. However, these functional modules obtained from bioinformatic analyses require experimental confirmation.
In conclusion, our study represents the first comprehensive analysis of a lncRNA-miRNA-mRNA network in ccRCC. The results revealed certain novel lncRNAs involved in ccRcc, such as DGCR5, MYCNOS, and PART1, which may be involved in the progression of ccRCC through cytochrome P450-mediated drug metabolism. These findings expand the existing knowledge on IncRNA characteristics and provide new tools for the identification of lcRNAs in ccRCC, which can help to elucidate the disease processes and identify new targets for therapy.

\section{Acknowledgements}

Not applicable.

\section{Funding}

The present study was supported by the National Natural Science Foundation of China (grant no. 81702495), the Natural Science Foundation of Anhui Province (grant no. KJ2018A0214), and the Anhui Natural Science Foundation (grant no. 1808085MH293).

\section{Availability of data and materials}

The datasets used and/or analyzed during the current study are available from the corresponding author on reasonable request.

\section{Authors' contributions}

BL and TM performed the data analyses and wrote the manuscript. QL, SW, WS and WL contributed significantly in data analyses and manuscript revision. JL and YG conceived and designed the study. All authors read and approved the final manuscript and agree to be accountable for all aspects of the research in ensuring that the accuracy or integrity of any part of the work are appropriately investigated and resolved.

\section{Ethics approval and consent to participate}

Not applicable.

\section{Patient consent for publication}

Not applicable.

\section{Competing interests}

The authors declare that they have no competing interests.

\section{References}

1. Siegel R, Ma J, Zou Z and Jemal A: Cancer statistics, 2014. CA Cancer J Clin 64: 9-29, 2014.

2. Gago-Dominguez M, Castelao JE, Yuan JM, Ross RK and Yu MC: Increased risk of renal cell carcinoma subsequent to hysterectomy. Cancer Epidemiol Biomarkers Prev 8: 999-1003, 1999.

3. Linehan WM: Genetic basis of kidney cancer: Role of genomics for the development of disease-based therapeutics. Genome Res 22: 2089-2100, 2012.

4. KapranovP,Cheng J,DikeS,Nix DA,DuttaguptaR, Willingham AT, Stadler PF, Hertel J, Hackermüller J, Hofacker IL, et al: RNA maps reveal new RNA classes and a possible function for pervasive transcription. Science 316: 1484-1488, 2007. 
5. Guttman M, Donaghey J, Carey BW, Garber M, Grenier JK, Munson G, Young G, Lucas AB, Ach R, Bruhn L, et al: lincRNAs act in the circuitry controlling pluripotency and differentiation. Nature 477: 295-300, 2011

6. Wilusz JE, Sunwoo H and Spector DL: Long noncoding RNAs: Functional surprises from the RNA world. Genes Dev 23: 1494-1504, 2009.

7. Bartel DP: MicroRNAs: Target recognition and regulatory functions. Cell 136: 215-233, 2009.

8. Farazi TA, Spitzer JI, Morozov P and Tuschl T: miRNAs in human cancer. J Pathol 223: 102-115, 2011.

9. Kwok ZH and Tay Y: Long noncoding RNAs: Lincs between human health and disease. Biochem Soc Trans 45: 805-812, 2017.

10. Crea F, Watahiki A, Quagliata L, Xue H, Pikor L, Parolia A, Wang Y, Lin D, Lam WL, Farrar WL, et al: Identification of a long non-coding RNA as a novel biomarker and potential therapeutic target for metastatic prostate cancer. Oncotarget 5: 764-774, 2014

11. Ebert MS and Sharp PA: Emerging roles for natural microRNA sponges. Curr Biol 20: R858-R861, 2010.

12. Arvey A, Larsson E, Sander C, Leslie CS and Marks DS: Target mRNA abundance dilutes microRNA and siRNA activity. Mol Syst Biol 6: 363, 2010 .

13. Tay Y, Rinn J and Pandolfi PP: The multilayered complexity of ceRNA crosstalk and competition. Nature 505: 344-352, 2014.

14. Panzitt K, Tschernatsch MM, Guelly C, Moustafa T, Stradner M Strohmaier HM, Buck CR, Denk H, Schroeder R, Trauner M and Zatloukal K: Characterization of HULC, a novel gene with striking up-regulation in hepatocellular carcinoma, as noncoding RNA. Gastroenterology 132: 330-342, 2007.

15. Cesana M, Cacchiarelli D, Legnini I, Santini T, Sthandier O, Chinappi M, Tramontano A and Bozzoni I: A long noncoding RNA controls muscle differentiation by functioning as a competing endogenous RNA. Cell 147: 358-369, 2011.

16. Bertozzi D, Iurlaro R, Sordet O, Marinello J, Zaffaroni N and Capranico G: Characterization of novel antisense HIF-1 $\alpha$ transcripts in human cancers. Cell Cycle 10: 3189-3197, 2011.

17. Frevel MA, Sowerby SJ, Petersen GB and Reeve AE: Methylation sequencing analysis refines the region of $\mathrm{H} 19$ epimutation in Wilms tumor. J Biol Chem 274: 29331-29340, 1999.

18. Chiesa N, De Crescenzo A, Mishra K, Perone L, Carella M, Palumbo O, Mussa A, Sparago A, Cerrato F, Russo S, et al: The KCNQ1OT1 imprinting control region and non-coding RNA: New properties derived from the study of Beckwith-Wiedemann syndrome and Silver-Russell syndrome cases. Hum Mol Genet 21: 10-25, 2012

19. Robinson MD, McCarthy DJ and Smyth GK: edgeR: A Bioconductor package for differential expression analysis of digital gene expression data. Bioinformatics 26: 139-140, 2010.

20. Cameron AC and Trivedi PK: Regression-based tests for overdispersion in the Poisson model. J Econ 46: 347-364, 1990.

21. Maritz JS and Lwin T: Empirical bayes methods with applications (Chapman \& Hall/CRC Monographs on Statistics \& Applied Probability) 2nd edition, 2018.

22. Solari A and Goeman JJ: Minimally adaptive BH: A tiny but uniform improvement of the procedure of Benjamini and Hochberg. Biom J 59: 776-780, 2017.

23. Koletsi D and Pandis N: Survival analysis, part 2: Kaplan-Meier method and the log-rank test. Am J Orthod Dentofacial Orthop 152: 569-571, 2017.

24. Jeggari A, Marks DS and Larsson E: miRcode: A map of putative microRNA target sites in the long non-coding transcriptome. Bioinformatics 28: 2062-2063, 2012.
25. Kanehisa M, Furumichi M, Tanabe M, Sato Y and Morishima K: KEGG: New perspectives on genomes, pathways, diseases and drugs. Nucleic Acids Res 45: D353-D361, 2016.

26. Wang W, Zhao E, Yu Y, Geng B, Zhang W and Li X: MiR-216a exerts tumor-suppressing functions in renal cell carcinoma by targeting TLR4. Am J Cancer Res 8: 476-488, 2018.

27. Ricketts CJ and Linehan WM: Gender specific mutation incidence and survival associations in clear cell renal cell carcinoma (CCRCC). PLoS One 10: e0140257, 2015.

28. Chen EG, Zhang JS, Xu S, Zhu XJ and Hu HH: Long non-coding RNA DGCR5 is involved in the regulation of proliferation, migration and invasion of lung cancer by targeting miR-1180. Am J Cancer Res 7: 1463-1475, 2017.

29. Dong HX, Wang R, Zeng J and Pan J: LncRNA DGCR5 promotes lung adenocarcinoma (LUAD) progression via inhibiting hsa-mir-22-3p. J Cell Physiol 233: 4126-4136, 2018.

30. Zhao X, Li D, Pu J, Mei H, Yang D, Xiang X, Qu H, Huang K, Zheng L and Tong Q: CTCF cooperates with noncoding RNA MYCNOS to promote neuroblastoma progression through facilitating MYCN expression. Oncogene 35: 3565-3576, 2016.

31. Malakootian M, Mirzadeh-Azad F, Naeli P, Fouani Y and Mowla SJ: A long non-coding RNA, PSORS1C3, located upstream of the human Oct4 gene is expressed in pluripotent and tumor cell lines. Modares J Med Sci Pathobiol 17: 105-117, 2014

32. Huang Y, Murakami T, Sano F, Kondo K, Nakaigawa N, Kishida T, Kubota Y, Nagashima Y and Yao M: Expression of aquaporin 1 in primary renal tumors: A prognostic indicator for clear-cell renal cell carcinoma. Eur Urol 56: 690-698, 2009.

33. Ma J, Zhou C, Yang J, Ding X, Zhu Y and Chen X: Expression of AQP6 and AQP8 in epithelial ovarian tumor. J Mol Histol 47: 129-134, 2016.

34. Liu F, Wu L, Wang A, Xu Y, Luo X, Liu X, Hua Y, Zhang D, Wu S, Lin T, et al: MicroRNA-138 attenuates epithelial-to-mesenchymal transition by targeting SOX4 in clear cell renal cell carcinoma. Am J Transl Res 9: 3611-3622, 2017.

35. Xiao H, Xiao W, Cao J, Li H, Guan W, Guo X, Chen K, Zheng T, Ye Z, Wang J and Xu H: miR-206 functions as a novel cell cycle regulator and tumor suppressor in clear-cell renal cell carcinoma. Cancer Lett 374: 107-116, 2016.

36. Fan Y, Ma X, Li H, Gao Y, Huang Q, Zhang Y, Bao X, Du Q, Luo G, Liu K, et al: miR-122 promotes metastasis of clear-cell renal cell carcinoma by downregulating Dicer. Int J Cancer 142: 547-560, 2018.

37. Huang J, Kong W, Zhang J, Chen Y, Xue W, Liu D and Huang Y: c-Myc modulates glucose metabolism via regulation of miR-184/PKM2 pathway in clear-cell renal cell carcinoma. Int J Oncol 49: 1569-1575, 2016.

38. Mitsui Y, Chang I, Fukuhara S, Hiraki M, Arichi N, Yasumoto H, Hirata H, Yamamura S, Shahryari V, Deng G, et al: CYP1B1 promotes tumorigenesis via altered expression of CDC20 and DAPK1 genes in renal cell carcinoma. BMC Cancer 15: 942, 2015.

39. Zaravinos A, Pieri M, Mourmouras N, Anastasiadou N, Zouvani I, Delakas D and Deltas C: Altered metabolic pathways in clear cell renal cell carcinoma: A meta-analysis and validation study focused on the deregulated genes and their associated networks. Oncoscience 1: 117-131, 2014.

(i) $\ominus$ This work is licensed under a Creative Commons Attribution-NonCommercial-NoDerivatives 4.0 International (CC BY-NC-ND 4.0) License. 